\title{
Cultura, cultura escolar y contenidos de enseñanza
}

\author{
José M. Esteve. Julio Vera. \\ José Terrón. Soledad Franco. \\ Amparo Civila.
}

\section{Un examen a la cultura escolar.}

Antes de comenzar a leer este escrito busque un papel y un lápiz y prepárese para contestar a un examen. Consta de diez preguntas y es muy fácil. Corresponde a un nivel de séptimo de E.G.B.; por tanto, usted, una persona culta, no debe tener ninguna dificultad en contestarlo: si los niños de 12 años consiguen aprobarlo, no esperamos menos de una persona como usted. Debemos advertirle, además, que el examen es real. Nuestro equipo de investigación no se lo ha inventado. Nos hemos limitado a recoger cientos de exámenes en diferentes colegios y a mezclar preguntas de distintas materias, respetando su redacción original. Como ya no es un niño no vamos a presionarle con el tiempo, tómese todo el que quiera, piense las respuestas y vaya escribiéndolas; pero si tarda demasiado, respire tranquilo y alégrese de no tener 12 años, porque ellos tienen que contestar lo mismo con el tiempo contado. ¿Está preparado? No haga trampas. Juegue contra sí mismo y ponga a prueba su capacidad de contestarlas. Al acabar puede contrastar sus respuestas, contabilizando aciertos y errores, con las soluciones que se ofrecen en una nota antes de la bibliografía. Sólo son diez preguntas:

1. ¿Qué se consiguió con la Paz de Augsburgo?

2. Define y pon un ejemplo de oración recíproca indirecta.

3. Escribe el número de caras, aristas y vértices del hexaedro.

4. ¿Qué clima corresponde a la sabana?

5. Escribe los principales biomas terrestres.

6. ¿Quién organiza el Congreso de Berlín de 1885? ¿Qué se decide?

7. ¿Cómo se llaman las células que producen los gametangios?

8. ¿Qué es una sinalefa?

9. Escribe los procesos mecánicos y químicos que tienen lugar en el intestino delgado. 
10. Características de las células eucarióticas.

¿Cuántas preguntas ha respondido con seguridad? ¿En cuántas de ellas reconoce no tener ni idea?

Ahora puede volver a alegrarse de no tener 12 años. Si los tuviera sería catalogado como fracasado escolar; pero usted puede respirar tranquilo: usted es un adulto que ya no depende de un examen; usted es un individuo razonablemente culto y deseoso de seguir cultivándose, como lo muestra el hecho de que, despreciando otras ocupaciones, se ponga a leer estas páginas; probablemente sea usted un padre o una madre medianamente contenta con la educación de sus hijos; y, desde luego, le supongo una persona equilibrada y razonable que aporta su trabajo a la vida social. ¿Cómo es posible, entonces, que fracase tan rotundamente en un examen de nuestra Educación General Básica? Probablemente el problema se situe en un error sobre los conceptos de "educación", de "general" o de "básica"; pero, ¿es usted consciente, sin embargo, de que los niños de 12 años pueden ser suspendidos y catalogados como fracasados escolares por no saberse lo mismo que usted tampoco sabe contestar?

¿Por qué presupongo que usted no ha aprobado el examen inicial? Pues verá, se nos ocurrió recoger exámenes de séptimo de E.G.B. y pasarlos a distintos grupos de alumnos en la Universidad. Los resultados obtenidos por 125 alumnos universitarios con el examen que encabeza estas páginas, corregido generosamente, les otorga una puntuación media de 2,48 puntos sobre $10, \mathrm{y}$ en ellos se acumulan 456 preguntas en blanco y 364 respuestas erróneas, frente a 198 respuestas acertadas y 232 respuestas incompletas; y tomando como criterio el 5 sólo consiguen aprobarlo 7 estudiantes universitarios de los 125 examinados.

\section{La selección de contenidos de la cultura escolar.}

Los alumnos de Barbiana, al escribir su Carta a una maestra (1975), acusan a los profesores de hacer trampas en los exámenes a los alumnos de las zonas rurales. Su acusación se basa en la distinta valoración de los maestros, dispuestos a considerar como algo imprescindible la distinción entre las fanerógamas y las criptógamas, mientras que el nombre de los árboles y el conocimiento de los cultivos que nos alimentan no tienen valor en la cultura escolar. Ellos argumentan que si se preguntara ésto en las escuelas, los hijos de los campesinos aprobarían, mientras que la mayor parte de la gente que nos consideramos cultos no conseguiríamos pasar los exámenes.

"Las dificultades deben ponerse en el mismo grado en que se encuentran en la vida. Si las ponéis con más fecuencia es que tenéis la manía de hacer la zancadilla. Como si estuvieseis en guerra con los niños. ¿Por qué lo hacéis? ¿Por su bien?" (p. 25).

A principios de la década de los ochenta un miembro de nuestro equipo formó parte de un tribunal para juzgar el acceso a la Universidad de los mayores de 25 años. Mientras vigilaba el desarrollo de la prueba escrita, propuso a sus colegas responder al cuestionario. El resultado fue el 
esperado: los tres miembros del tribunal, respetados profesores universitarios, no consiguieron aprobar. Nos planteamos entonces una duda que aún hoy nos interroga: ¿cómo podemos pedir, para entrar en la Universidad, un examen que sus catedráticos son incapaces de contestar?

Nuestro intento de responder a esa pregunta nos llevó a interrogarnos sobre el concepto de cultura, sobre los criterios con los que se elaboran los exámenes que, como señala Lobrot (1976), son el elemento clave del actual sistema educativo, y sobre el concepto de cultura escolar.

Llamamos cultura escolar a los conocimientos y contenidos que han sido seleccionados, del ámbito más amplio de la cultura general, para ser transmitidos en nuestras escuelas. Dentro del marco conceptual y valorativo de la cultura escolar, se toman luego las decisiones sobre los contenidos del curriculum. Estas dos ideas son muy simples; pero, provisionalmente, nos permiten entendernos para estudiar la relación entre el concepto general de cultura y los oscuros mecanismos con los que se selecciona lo que los niños $\mathrm{y}$ adolescentes deben estudiar en nuestras instituciones educativas.

$\mathrm{Al}$ estudiar esta relación entre cultura y cultura escolar, surgen varios hechos evidentes sobre los que vale la pena reflexionar.

El primero son los sesgos nacionalistas de la cultura escolar. En efecto, los libros de texto que se utilizan en nuestras escuelas seleccionan distintos contenidos en diferentes naciones. Para entender ésto, basta con preguntarle a un español culto por Goethe, por Balzac o por Joyce. Apenas si somos capaces de recordar un par de generalidades de cada uno de ellos. Entonces nos daremos cuenta de que ser una persona culta es algo diferente en cada país, y requiere el dominio de contenidos diferentes; ya que, en cada uno de nuestros sistemas escolares, hemos estudiado a figuras literarias de cuarta $\mathrm{o}$ de quinta fila, dudosas incluso en el ámbito de nuestra cultura, por el mero hecho de ser compatriotas; mientras que apenas hemos estudiado a grandes figuras de la literatura universal, por tratarse de escritores alemanes, franceses o irlandeses. Si estudiamos el mismo problema en los libros de Historia, encontraremos una versión distinta de los sesgos nacionalistas; ya que, al estudiar el mismo hecho histórico en los libros de texto de dos países diferentes llegaremos a dudar de que ambos puedan estar hablando de lo mismo. Como ejemplos más evidentes, podemos citar los enfoques de los libros de texto españoles e ingleses sobre el asunto de la Armada Invencible, o las versiones de los libros de texto belgas y españoles sobre la actuación de los Tercios de Flandes. En este tema vale la pena resaltar el hecho de que el furibundo nacionalismo de los libros de Historia de hace veinte años se ha difuminado en las últimas ediciones, probablemente porque alguien se ha dado cuenta de que el proyecto de unidad europea será más difícil si nuestros alumnos siguen estudiando textos en los que se nombra a Inglaterra como "la pérfida Albión", o en los que se incluyen comentarios como el siguiente:

"En realidad, Portugal estaba llamado a unir para siempre sus destinos a los de España y formar con ella una sola nación. Común fue su origen, numerosos los nexos familiares entre los monarcas de los dos países, y 
similares las aúreas páginas escritas por ambos en los ámbitos del mundo. Hasta la misma geografía de la península ibérica parece exigir esa fusión. Pero hay enemigos permanentes de ella; siempre lo han sido Inglaterra, Francia y Holanda" (Edelvives, 1963, p. 192).

El segundo problema que me plantea la cultura escolar se refiere a la utilidad de los contenidos. Peters (1977) considera que el aprendizaje de un contenido de enseñanza no puede considerarse educativo más que cuando, en torno a ese contenido, el alumno es capaz de desarrollar esquemas conceptuales propios: "El hombre educado debe poseer también algún conjunto de conocimientos y algún esquema conceptual que eleve esos conocimientos por encima del nivel de una serie de datos inconexos. Lo cual implica conocer los principios para poder organizar los datos... La persona educada debe tener también alguna comprensión del "porqué" de las cosas" (p. 39). En la misma línea, los más diversos autores coinciden en señalar que la cultura es algo que nos debe hacer capaces de entender el mundo que nos rodea. Sin embargo, al estudiar el contenido de la mayor parte de los libros escolares y los subsiguientes enfoques de los profesores, observamos que la cultura escolar selecciona preferentemente clasificaciones, enumeraciones, vocabularios básicos y convenciones científicas que no nos permiten entender la realidad, y que sólo tienen valor en el mundo escolar. Así, por ejemplo, hemos memorizado que el cuarzo cristaliza en el sistema hexagonal, tiene brillo vítreo y fractura concoide, y nos sentimos cultos por recordar su grado de dureza en la escala de Mohs; pero, ridículamente, no tenemos la menor idea de cómo pueda ser una fractura concoide, y si nos sitúan ante una formación rocosa, somos incapaces de distinguir el cuarzo de cualquier otro mineral. De la misma forma, hemos estudiado una larga lista de clasificaciones botánicas, entre las que se encuentra la exacta distinción entre las plantas cormofitas, talofitas y briofitas; pero somos incapaces de distinguir al olmo de la encina, o al haya del castaño; y no sabríamos diferenciar un sembrado de patatas de otro de zanahorias. Nos encontramos, por tanto, con un problema de significación de los contenidos aprendidos, y con un criterio que nos puede ayudar a discutir sobre la significación de estos contenidos. Este criterio podría formularse afirmando que cualquier contenido de enseñanza, para ser considerado valioso, debe concluir en el desarrollo de un esquema conceptual propio por parte de la persona que aprende, de forma tal que ésta sea capaz usar los contenidos aprendidos para entender una parte de la realidad, ayudándole así a entenderse a sí mismo o a entender el mundo que le rodea; sin embargo, con excesiva frecuencia, los contenidos de enseñanza carecen de toda relación con la realidad: se aprenden como parte de una cultura escolar en la que la única justificación de los contenidos consiste en repetirlos en los exámenes, después ya no tienen valor y pueden olvidarse. Naturalmente, con este esquema, el valor del aprendizaje a medio o largo plazo es prácticamente nulo, ya que no existen relaciones con la realidad que posibiliten su pervivencia. Así, la cultura escolar entretiene el tiempo de sus alumnos sin que éstos lleguen a entender el mundo que les rodea, ni sean capaces de encontrar relaciones entre la realidad y lo que aprenden en nuestras escuelas (Pérez, 1988).

El tercer problema se refiere a la selección de contenidos. Para explicarlo 
recurriremos a las preocupaciones de Guillermo Brown. En una de sus aventuras, el personaje de los cuentos infantiles se lamenta de no haber nacido en el siglo XVII. Ante la extrañeza de su interlocutor, el niño responde, con impecable lógica, que si hubiera nacido en esa fecha se ahorraba estudiar tres siglos de Historia. Pues bien, si analizamos la cultura escolar descubriremos la presencia agobiante de contenidos cuya permanencia en la escuela no se justifica más que por la tradición o la costumbre (Westoby, 1988; Pérez, 1998), mientras que otros contenidos que nos permitirían comprender el mundo contemporáneo no tienen cabida, o se integran de forma muy lenta a la cultura escolar. En el fondo de esta cuestión está la necesidad de revisar periódicamente los contenidos de la educación, integrando nuevos conocimientos, nuevos enfoques y nuevos planteamientos científicos. En otras ocasiones, el problema estriba en añadir al estudio de los principios generales un apartado que nos permita entender las aplicaciones prácticas más importantes de los conocimientos científicos que estudiamos, favoreciendo así la comprensión de las aplicaciones tecnológicas más usuales, con las que funciona la vida cotidiana en el mundo que nos rodea. Sin embargo, esta petición plantea el problema de que no puede seguirse, indefinidamente, sumando nuevas exigencias sobre los jóvenes estudiantes, sin, al mismo tiempo, eliminar contenidos irrelevantes. La discusión sobre qué contenidos puedan considerarse irrelevantes debe hacerse muy despacio, atendiendo a criterios encontrados, y contando con la resistencia conservadora de los profesores que los imparten, dispuestos siempre a considerar un agravio que se pueda discutir sobre la relevancia de unos conocimientos que constituyen la razón de ser de su actividad profesional. Recientemente, he tenido la ocasión de conocer el trauma que supuso en su día la desaparición de la Caligrafía como materia de enseñanza en las Escuelas Normales de Magisterio, y soy capaz de imaginarme las reacciones de los profesores que enseñaban esa materia al quedarse sin trabajo; de la misma manera, es de esperar reacciones agresivas por parte de los profesores a los que se les dice que deben modificar el enfoque o los contenidos de la materia que han venido explicando desde siempre, abandonando temas que llevan explicando toda su vida profesional, e incorporando otros de los que ni siquiera se hablaba cuando ellos concluyeron sus estudios (Sarason, 1982). Insisto en que la discusión sobre la relevancia de los contenidos debe hacerse con mucha prudencia y tras un sereno diálogo con todas las partes implicadas; pero tengo absolutamente claro que no tiene sentido que un hombre culto del siglo XXI estudie tres años de Física y, al mismo tiempo, utilice cotidianamente un teléfono, un ordenador y un televisor sin la menor idea de cómo funcionan. Para considerarme culto, me gustaría entender cómo se transmite la voz y las imágenes de televisión, cómo procesa la información un chip y por qué levantan el vuelo los aviones. Sin embargo, ninguno de estos contenidos, ni otros semejantes que nos permitirían entender el mundo en que vivimos, se han incorporado a la cultura escolar; y ésto sólo en el ámbito de la tecnología, ya que cotidianamente oímos hablar del aumento de la inflación, o del P.I.B., sin que se considere importante integrar el estudio de los conceptos básicos de la Economía en el curriculum escolar. De la misma forma, la Informática se ha convertido en un contenido imprescindible para encontrar un empleo en la sociedad contemporánea: desde la más pequeña oficina a las grandes corporaciones internacionales la mayor parte de las empresas utilizan la informática como un elemento de trabajo 
imprescindible, sin que hasta el presente nuestro sistema educativo haya sido capaz de responder a esta demanda social incorporando unos contenidos mínimos de informática en la formación básica de nuestros ciudadanos. Se considera que los horarios están saturados de contenidos y que no hay espacio para incorporar nuevas materias; sin embargo, nuestro estudio pone de manifiesto la presencia de grandes espacios de contenidos irrelevantes, que, ni ayudan al alumno a entender el mundo que le rodea, ni tienen pervivencia a medio o largo plazo.

\section{La significación y el valor educativo de los contenidos.}

Una cuarta cuestión hace referencia a la significación de los contenidos. Para explicar ésto volveré a algunos ejemplos del examen inicial. La primera pregunta: ¿Qué se consiguió con la Paz de Augsburgo?, sólo fue contestada de forma correcta por 2 de los 125 estudiantes universitarios examinados, y cuando se plantea a otros grupos de personas con un nivel de estudios universitarios el número de aciertos es igualmente bajo. Sin embargo, ésto no quiere decir que esas personas no sepan nada de los acontecimientos relacionados con la Paz de Augsburgo. En efecto, si se les pregunta sobre las guerras de religión entre católicos y protestantes, es decir sobre el proceso que culmina con la Paz de Augsburgo, una abrumadora mayoría es capaz de situar los acontecimientos en la época histórica correspondiente y en el marco geográfico adecuado; incluso, son capaces de valorar su significado histórico y la actuación de algunos de los personajes implicados en ellas. El problema está en que, simplemente, han olvidado que aquel proceso histórico concluyera en un tratado que recibió el nombre de Paz de Augsburgo. Igual ocurre si analizamos la pregunta mejor contestada del examen: ¿Qué es una sinalefa? De 125 alumnos, 37 la contestan adecuadamente; pero, en realidad, casi el cien por cien de los alumnos universitarios saben que, a la hora de medir un verso, cuando una palabra termina en vocal y la siguiente también empieza por vocal ambas se unen contabilizando una sola sílaba. Si 88 alumnos dejan la respuesta en blanco o comenten un error en la definición, es simplemente porque han olvidado que esa regla métrica se llama sinalefa. Por tanto, el elevado porcentaje de error en esta pregunta no quiere decir que los universitarios sean incapaces de medir un verso, sino exclusivamente que han olvidado el término correspondiente.

La conclusión que sacamos de estos ejemplos es que, en muchos casos, los errores en las respuestas se corresponden con la forma en que se enfocan los conocimientos y se hacen los exámenes; ya que en éstos se prefieren las cuestiones que se refieren a vocabularios, definiciones cortas, clasificaciones y convenciones, antes que las preguntas que exigen la aplicación, la comprensión o la valoración de los conocimientos adquiridos. A partir de esta idea, nuestra investigación ha procurado profundizar en dos hechos desgraciadamente frecuentes en nuestra enseñanza: la acumulación de conocimientos desprovistos de significado; y el sesgo en la elección de las preguntas para los exámenes.

La acumulación de conocimientos desprovistos de significado puede estudiarse claramente en dos cuestiones en las que nuestros estudiantes 
universitarios fracasan clamorosamente.

La primera consiste en enseñarles un mapa de España con una borrasca centrada en el Atlántico, entre Portugal y Galicia, tal como se les presenta cada día en televisión, en los espacios dedicados a las predicciones metereológicas. El mapa se acompaña del siguiente enunciado:

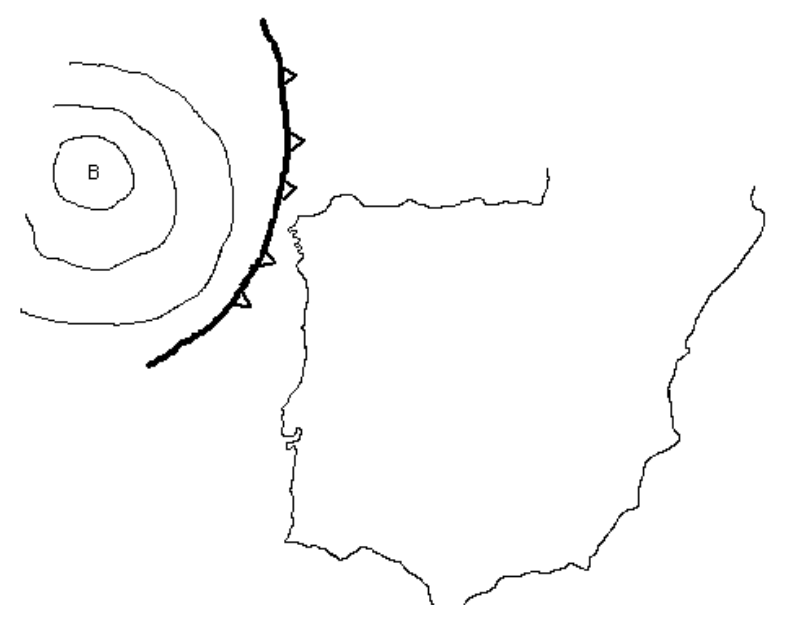

Como usted puede ver diariamente en televisión, este mapa representa una borrasca situada al oeste de la península Ibérica.

1. ¿qué ocurre más frecuentemente?

a. Que la borrasca avance hacia el este afectando a la península.

b. Que la borrasca se pierda en el Atlántico avanzando hacia el oeste.

c. Que la borrasca evolucione en el sentido norte-sur.

2. Explique la razón de su respuesta a la pregunta 1.

El análisis de las respuestas nos permite observar que todos los estudiantes responden a la primera pregunta afirmando que la borrasca se desplazará en dirección este, afectando a la península. Sus respuestas se basan en su experiencia cotidiana de los mapas de televisión, ya que es extraordinariamente raro encontrar un alumno que sepa responder a la segunda pregunta. En ella se ofrecen las más disparatadas razones para explicar la pertinaz tendencia de las borrascas, en nuestra latitud, a perseverar en la dirección oeste-este. Sin embargo, los alumnos disponen de los conocimientos necesarios para dar una respuesta correcta. En efecto, si se les pregunta por los tipos de vientos que conocen, inmediatamente citan la existencia de los vientos alisios y contra-alisios, el problema está en que sólo han aprendido sus nombres; pero, de ninguna manera, la forma en que funcionan o las razones de su sistema de funcionamiento. Sin embargo, en una ocasión, un alumno universitario no sólo supo responder adecuadamente a la pregunta sobre la razón de que las borrascas se desplacen en dirección 
oeste-este, sino que además fue capaz de relacionar con este hecho la salida de Colón hacia América, descendiendo hasta las islas Canarias y volviendo por las Azores hacia el norte de Portugal, y el hecho de que los vuelos transatlánticos entre América y Europa tengan una duración menor que en el trayecto de ida por el aprovechamiento del Jet Stream. El alumno acabó con un comentario de agradecimiento hacia su antiguo profesor, diciendo que era un hombre al que "le gustaba relacionar". Su respuesta nos permitió entender el problema de sus compañeros: al parecer sus profesores sólo se habían preocupado de hacerles memorizar contenidos que no saben cómo usar y que no tienen relación con ningún otro hecho estudiado, ni con ningún acontecimiento de su vida cotidiana. Lamentablemente, las aportaciones del constructivismo siguen sin ser aplicadas por la mayoría de nuestros profesores a su práctica educativa, permitiendo la pervivencia de hechos como los descritos (Barrón, 1991; Benlloch, 1984; Coll, 1987, 1990; Driver, 1988,1989; Pozo, 1987, 1990; Sebastiá, 1989). La pregunta es evidente: ¿somos más cultos por repetir de memoria que existen unos vientos llamados alisios y contra-alisisos, cuando somos incapaces de entender los mapas del tiempo que estamos viendo en televisión todos los días?

La segunda cuestión que voy a utilizar para profundizar en el problema de la acumulación de conocimientos desprovistos de significado es muy simple. No hay más que preguntar a un grupo de estudiantes universitarios por qué hace más calor en verano que en invierno. Se supone que ésta es una pregunta básica, cotidiana, a la que tendríamos que dar una respuesta clara y sin dudas; pues bien, no llega al $3 \%$ el número de universitarios que es capaz de contestarla; y lo que es más grave, si se les enseña el mapa que aparece a continuación, una abrumadora mayoría responde con el disparate de que al describir la Tierra una elipse alrededor del Sol, cuando la Tierra está más cerca hace más calor y es verano, y cuando está más lejos hace más frío y es invierno. Si en ese momento les señalamos que, de ser cierta su respuesta, tendríamos dos veranos y dos inviernos, la mayoría reconoce su incapacidad para explicar un hecho tan simple; mientras que otros aún se complican más, recordando que durante el invierno del hemisferio norte es verano en el hemisferio sur, hasta descubrir que tampoco son capaces de dar una explicación satisfactoria de este hecho.

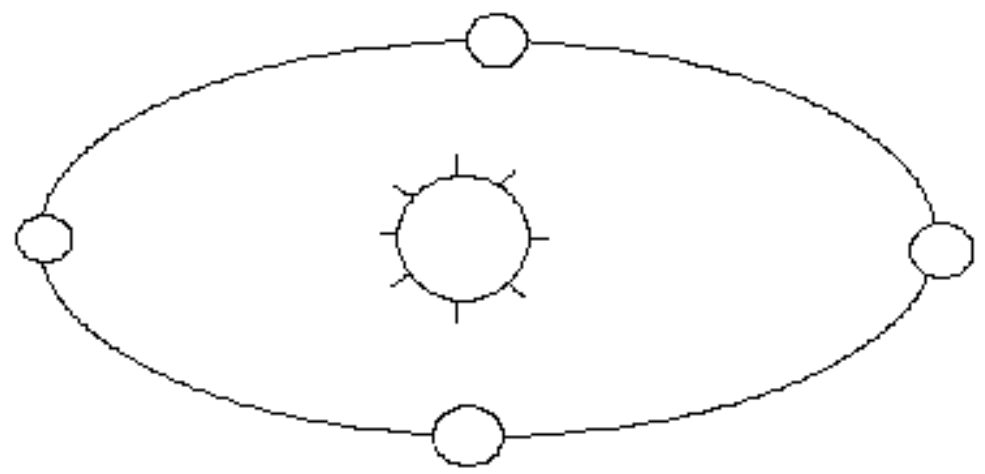

Sólo cuando se les explica sobre este gráfico la distinta incidencia de los rayos del sol en uno u otro hemisferio, debida a la inclinación del eje de la Tierra, comprenden por qué hace más calor en verano que en invierno; y, al 
mismo tiempo, por qué el verano de un hemisferio coincide con el invierno del otro y por qué en un polo hay seis meses de noche perpetua y, luego, seis meses de luz permanente.

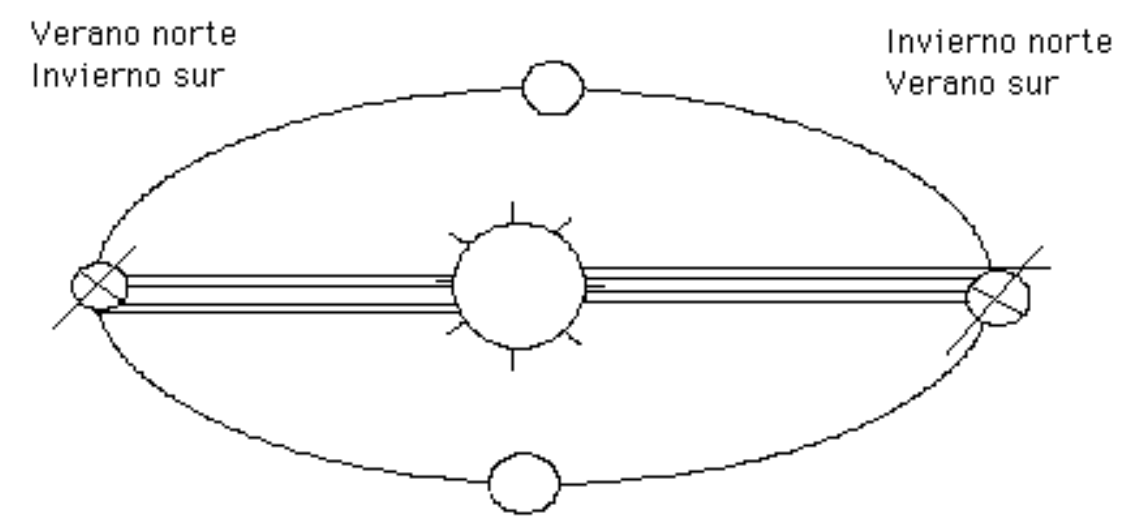

La idea de que la energía solar se distribuye en una superficie mayor o menor debido a la distinta inclinación de los rayos solares no se ha transmitido en las explicaciones que recibieron durante su escolaridad, hasta el punto de que la presentación del gráfico siguiente supone una auténtica revelación que les permite entender, en la Universidad, por fin y a sus veintidos años, por qué hace más calor en verano que en invierno.

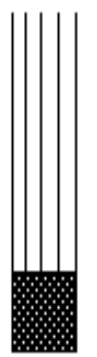

A

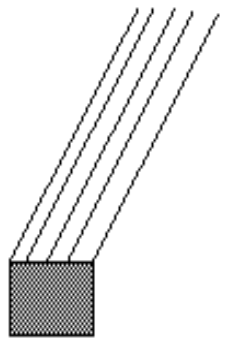

B

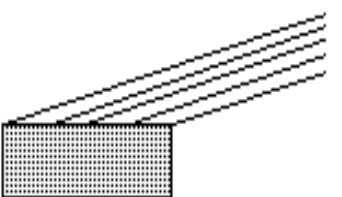

C

Cuanto más aumenta la inclinación de los rayos solares más disminuye la cantidad de calor recibida por unidad de superficie. De $\mathrm{A}$ a $\mathrm{C}$ el mismo número de calorías se distribuye sobre una superficie cada vez mayor.

La conclusión es evidente: la mayor parte de nuestros profesores no transmite los contenidos de enseñanza como una forma de entender el mundo que nos rodea, sino como datos aislados en los que el estudiante aprende hechos, clasificaciones, definiciones y convenciones que luego no sabe cómo utilizar. El estudiante se limita a memorizarlos hasta llegar al momento del examen y luego los olvida, ya que los contenidos aprendidos carecen de fuerza explicativa para entender algún aspecto de la realidad, y nadie se ha preocupado de desarrollar la aplicación de los contenidos aprendidos, estableciendo las relaciones pertinentes entre la cultura escolar y el mundo que nos rodea (Pozo, 1987). Así, es frecuente que muchos alumnos universitarios, al preguntarles por qué hace más calor en verano que en invierno, sean capaces de responder con seguridad que la razón es la inclinación del eje de la Tierra, sin ser luego capaces de dar una explicación 
razonada de cómo influye la inclinación del eje de la Tierra en el aumento de las temperaturas.

El estudio de estos errores conceptuales (misconceptions), en los que se detecta la falta de comprensión de un tema enseñado a grandes masas de estudiantes, es una de las vías más prometedoras de avance en la investigación de las distintas Didácticas Especiales, que tienen en él un elemento primordial para diagnosticar la calidad de la enseñanza que se imparte en sus respectivas materias y para mejorar las estrategias de enseñanza que se vienen empleando (Anderson, 1990; Carrascosa, 1985,1987; Furió, 1986,1987; Gil, 1987, 1991; Llorents, 1989; Ross, 1991; Stavy, 1991). Igualmente, puede ser de un extraordinario valor para mejorar la calidad de nuestra formación inicial de profesores, ya que, muy frecuentemente, no revelan más que la falta de comprensión de nuestros maestros sobre los temas en los que se detectan esos errores conceptuales (Banerjee, 1991; Hewson y Hewson, 1987, 1988; Johnston y Scott, 1991).

\section{Los sesgos en la elaboración de los exámenes: consecuencias educativas.}

El otro gran problema que afecta a la significación de los contenidos aprendidos es el sesgo en la elección de las preguntas de examen, ya que el tipo de preguntas que se hacen en los exámenes es un elemento determinante de la forma de estudiar de los alumnos. En efecto, es bien sabido que los alumnos que van a enfrentarse a un examen de respuestas de elección múltiple seleccionan y dan prioridad en su estudio a los elementos susceptibles de ser utilizados en este tipo de preguntas; es decir, secuencias de contenidos cortas en las que priman datos, cifras, definiciones, clasificaciones o convenciones que pueden preguntarse y responderse de forma breve y objetiva. Por tanto, este tipo de exámenes excluye frecuentemente las preguntas de aplicación, o las que exigen una valoración crítica o una síntesis general de procesos complejos. De hecho, al estudiar los exámenes que habíamos recogido en los centros escolares enseguida tuvimos conciencia de que predominaban las preguntas cuya contestación se reducía al ámbito de la memorización, y nos propusimos elaborar una tabla de frecuencias clasificando una a una las preguntas contenidas en todos los exámenes recogidos siguiendo la vieja taxonomía de Bloom y Krathwohl (1972).

Las taxonomías de los objetivos de la educación sufrieron una fuerte contestación a principios de los años ochenta (Gimeno, 1982), al desprenderse de ellas un hacer pedagógico obsesionado por la eficiencia y gobernado por programaciones milimétricas; sin embargo, vale la pena no perder de vista el origen de dichas taxonomías, que surgen de una "comisión de examinadores de enseñanza técnica y universitaria" que buscaba "la creación de un entramado teórico que facilitara los cambios de impresiones entre los examinadores... para impulsar el intercambio de los materiales de las pruebas y de las ideas entorno a ellas,... estimular los trabajos de investigación sobre las pruebas de los exámenes y sobre las relaciones entre los examinadores y los alumnos" (Bloom, 1972, p. 16). Por ello, nuestro equipo de investigación consideró más prudente clasificar las preguntas de 
los exámenes que estábamos estudiando siguiendo una taxonomía conocida, muy meditada y elaborada durante años por un amplio grupo de expertos, antes que emprender el dudoso trabajo de elaborar una nueva taxonomía.

Como se recordará, el equipo de Bloom utiliza seis categorías básicas, que distinguen en el aprendizaje del alumno seis operaciones diferentes: memorizar, comprender, aplicar, analizar, sintetizar y valorar los contenidos aprendidos. Utilizando estas seis categorías elaboramos la correspondiente tabla de frecuencias descubriendo la presencia de cada una de ellas sobre el total de las preguntas que los profesores utilizan en sus exámenes. En muchas ocasiones el sesgo proviene de las concepciones del profesor sobre su materia de enseñanza, dando prioridad a la memorización sobre el resto de actividades que pueden desarrollar los alumnos.

\section{El profesor como maestro de humanidad: una visión educativa de los contenidos de enseñanza.}

El camino y la meta me los marcó Unamuno en una necrológica de Giner de los Ríos, leída por azar en el Boletín de la Institución Libre de Enseñanza:

"Era tan hombre y tan maestro, y tan poco profesor -el que profesa algo-, que su pensamiento estaba en continua y constante marcha, mejor aun, conocimiento... y es que no escribía lo ya pensado, sino que pensaba escribiendo como pensaba hablando, pensaba viviendo, que era su vida pensar y sentir y hacer pensar y sentir".

..."Era su vida pensar y sentir y hacer pensar y sentir"... Miguel de Unamuno y su preocupación por enlazar pensamiento y sentimiento... Nunca encontré una mejor definición del magisterio: dedicar la propia vida a pensar y sentir, y a hacer pensar y sentir; ambas cosas juntas. La misma idea se repite en la descripción que hacen de su actividad varios maestros: Díez (1998) expresa así su visión de la enseñanza: "ahora entiendo la escuela como un sitio adonde vamos a aprender, donde compartirmos el tiempo, el espacio y el afecto con los demás; donde siempre habrá alguien para sorprenderte, para emocionarte, para decirte al oído algún secreto magnífico". Corbalán (1998), tras afirmar que el maestro en clase tiene que divertirse, buscar el ansia de saber y propiciar una atmósfera de investigación, concluye: "Y no se piense que sólo se abre la mente a los alumnos. También la del profesor se expande y se llena de nuevos matices y perspectivas más amplias, y funciona la relación enriquecedora en los dos sentidos. Mi experiencia, al menos, me dice que algunos de los juegos y problemas con los que he disfrutado, y que sigo utilizando, han tenido su origen en la dinámica de la clase... Y cuando se crea esa atmósfera mágica en clase, con los fluídos intelectuales en movimiento, pocas actividades hay más placenteras".

Hace tiempo, descubrí que el objetivo último es ser maestro de humanidad. Lo único que de verdad importa es ayudarles a comprenderse a sí mismos y a entender el mundo que les rodea. Para ello, no hay otro camino que rescatar, en cada una de nuestras lecciones, el valor humano del conocimiento. Todas las ciencias tienen en su origen a un hombre o una mujer preocupados por desentrañar la estructura de la realidad. Alguien, 
alguna vez, elaboró los conocimientos del tema que explicas, como respuesta a una preocupación vital. Alguien, sumido en la duda, inquieto por una nueva pregunta, elaboró los conocimientos del tema que mañana te toca explicar. Y ahora, para hacer que tus alumnos aprendan la respuesta, no tienes otro camino más que rescatar la pregunta original. No tiene sentido dar respuestas a quienes no se han planteado la pregunta; por eso, la tarea básica del docente es recuperar las preguntas, las inquietudes, el proceso de búsqueda de los hombres y mujeres que elaboraron los conocimientos que ahora figuran en nuestros libros. La primera tarea es crear inquietud, descubrir el valor de lo que vamos a aprender, recrear el estado de curiosidad en el que se elaboraron las respuestas. Para ello hay que abandonar las profesiones de fe en las respuestas ordenadas de los libros, volver las miradas de nuestros alumnos hacia el mundo que nos rodea y rescatar las preguntas iniciales obligándoles a pensar.

Cada día, antes de explicar un tema, necesito preguntarme qué sentido tiene el que yo me ponga ante un grupo de alumnos para hablar de esos contenidos, qué les voy a aportar, qué espero conseguir. Y luego, cómo enganchar lo que ellos saben, lo que han vivido, lo que les puede preocupar, con los nuevos contenidos que voy a introducir. Por último me lanzo un reto: me tengo que divertir explicándolo, y ésto es imposible si cada año repito la explicación del tema como una salmodia, con la misma gracia en el mismo sitio y los mismos ejemplos; llevo veinticinco años oyéndome explicar los temas, en algunas ocasiones, repitiéndolos dos o tres veces en distintos grupos; he calculado que me jubilo el año $2.021 \mathrm{y}$ estoy seguro de que moriré de aburrimiento si me oigo año tras año repitiendo lo mismo, con mis papeles cada vez más amarillos y los rebordes carcomidos. La renovación pedagógica, para mí, es una forma de egoísmo: con independencia del deseo de mejorar el aprendizaje de mis alumnos, la necesito como una forma de encontrarme vivo en la enseñanza, como un desafío personal para investigar nuevas formas de comunicación, nuevos caminos para hacer pensar a mis alumnos... "pensaba hablando, pensaba viviendo, que era su vida pensar y sentir y hacer pensar y sentir..." Desde esta perspectiva, la enseñanza recupera cada día el sentido de una aventura que te rescata del tedio y del aburrimiento, y entonces encuentras la libertad de expresar en clase algo que te es muy querido. Inmediatamente recibes la respuesta: cien alumnos pican el anzuelo de tu palabra y ya puedes dejar correr el sedal, modulas el ritmo de tu explicación a la frecuencia que ellos emiten con sus gestos y sus preguntas, y la hora se pasa en un suspiro -también para ellos-. Y entonces descubres la alegría: ese momento de magia te recompensa las horas de estudio y te hace sentirte útil en la enseñanza.

No hay mejor regalo de los dioses que encontrar un maestro. A veces tenemos la fortuna de encontrar a alguien cuya palabra nos abre horizontes antes insospechados, nos enfrenta con nosotros mismos rompiendo las barreras de nuestras limitaciones; su discurso rescata pensamientos presentidos que no nos atrevíamos a formular, e inquietudes latentes que estallan con una nueva luz. Y, curiosamente, no nos sentimos humillados por seguir el curso de un pensamiento ajeno; por el contrario, su discurso nos libera y nos ensancha creando en nosotros un juicio paralelo con el que reestructuramos nuestra forma de ver la realidad; y luego, extinguida la 
palabra, aún encontramos los ecos que rebotan en nuestro interior obligándonos a ir más allá, a pensar por nuestra cuenta, a extraer nuevas conclusiones que no estaban en el discurso original... Este es el objetivo: ser maestros de humanidad... a través de las materias que enseñamos, o quizás, a pesar de las materias que enseñamos; recuperar y transmitir el valor de la sabiduría; rescatar para nuestros alumnos, de entre la maraña de la ciencia y la cultura, el valor de las preguntas fundamentales que han preocupado al hombre en los momentos claves de la Historia, permitiéndoles así enterderse a sí mismos y comprender el mundo que les rodea.

Nota: Respuestas a las preguntas del examen inicial. 1-. El fin de las guerras religiosas en Europa entre católicos y protestantes. 2-. Es aquella en que los sujetos realizan y reciben a la vez una acción, y el pronombre recíproco es el C.I.. Ej. Juan y María se besan. 3-. 6 caras, 12 aristas y 8 vértices. 4-. Clima tropical o ecuatorial. 5-. Lugar diferenciado donde se desarrolla la vida: tundra, estepa, selva, sabana, desierto... 6.- Organizado por Alemania (Bismarck) para el reparto de Africa entre las potencias europeas. 7.Gametos (células sexuales o reproductoras). 8.- La unión de una vocal con otra, formando una sola sílaba métrica, cuando en el mismo verso la última sílaba de una palabra termina en vocal y la primera de la palabra siguiente comienza también por vocal. 9.- Se segregan los jugos gástricos (jugos pancreáticos) que descomponen el alimento y las vellosidades intestinales absorben las sustancias aprovechables. 10.- Son aquellas células que poseen membrana, citoplasma y núcleo bien diferenciados, el núcleo está separado del citoplasma por una membrana celular.

\section{Bibliografía}

Aguirre, L. (1985) Los adolescentes y el aprendizaje de las ciencias. Madrid, Brevarios de Educación, MEC.

Aliberas, J., Gutiérrez, R. e Izquierdo, M. (1989) Modelos de aprendizaje en la didáctica de las ciencias.Investigación en la Escuela, 9, pp.17-24.

Alumnos de Barbiana. (1975) Carta a una maestra. Barcelona, Nova Terra.

Anderson, B. (1990) Pupils' conception of matter and its transformations (age 12-16). Studies in Science Education, 18, pp. 53-58.

Astolfi, J.P. y Develay, M. (1989) La didactique des sciences . París, PUF.

Ausubel, D.P., Novak, J.D. y Hanesian, H. (1973) Psicología educativa. Un punto de vista cognoscitivo. México, Trillas.

Aznar, P. y otros. (1992) Constructivismo y educación. Valencia, Tirant lo Blanch.

Banerjee, A. (1991) Misconceptions of students and teachers in chemical equilibrium. International Journal of Science Education, 13, pp. 487-494.

Barrón, A. (1991) Aprendizaje por descubrimiento. Análisis crítico y 
reconstrucción teórica. Salamanca, Amarú.

Benlloch, A. (1984) Por un aprendizaje constructivista de las ciencias. Madrid, Visor. Camaño, A., Mayos, C., Maestre, G. y Ventura, T. (1983) Consideraciones sobre algunos errores conceptuales en el aprendizaje de la Química en el Bachillerato.Enseñanza de las Ciencias. 1, 3, pp. 198-200.

Bernstein, B. (1990) Poder, educación y conciencia. Sociología de la transmisión cultural Barcelona, El Roure.

Bloom, B. S. (ed.) (1972) Taxonomía de los objetivos de la educación. Clasificación de las metas educativas. Ambito del conocimiento. Alcoy, Marfil.

Bruner, J.S. (1985) Cultura, políticos y pedagogía. Cuadernos de Pedagogía, 121, enero, pp. 7-10.

Caivano, F. y Carbonell, J. (1983) Escuela, cultura, territorio. Cuadernos de Pedagogía, 102, junio, pp.4-6.

Carrascosa, J. (1985) Errores conceptuales en la enseñanza de la Física y Química: una revisión bibliográfica.Enseñanza de lss Ciencias. 3, 3, pp. 230-234.

Carrascosa, J. (1987) Tratamiento didáctico en la enseñanza de las ciencias de los errores conceptuales. Tesis doctoral. Facultad de Químicas de la Universidad de Valencia.

Coll, C. (1987) Significado y sentido en el aprendizaje escolar. Reflexiones en torno al concepto de aprendizaje significativo.Infancia y Aprendizaje, 41, pp. 131-142.

Coll, C. (1990) Aprendizaje escolar y construcción del conocimiento. Barcelona, Paidós. Cubero, R. (1989) Cómo trabajar con las ideas de los alumnos. Sevilla, Diada.

Corbalán, F. (1998) "Una vida enseñando matemáticas". Cuadernos de Pedagogía. 266, feb., pp. 72-75.

Díez, M.C. (1998) "El oficio del maestro es aprender".Cuadernos de Pedagogía. 266, feb., pp. 58-61.

Driver, L.R. (1988) Un enfoque constructivista para el desarrollo del currículo en ciencias. Enseñanza de las Ciencias, 6, 2, pp. 109-120.

Driver, R., Guesne, E. y Tiberghien, A. (1989) Ideas cientificas en la infancia y en la adolescencia. Madrid, Morata -MEC.

Edelvives. (1963) Historia Universal. Zaragoza, Editorial Luis Vives.

Fernández, E. (1979) Estructura y didáctica de las ciencias . Madrid, MEC. 
Freinet, C. (1976) La enseñanza de las ciencias . Barcelona, Laia.

Furió, C. (1986) Metodologías utilizadas en la detección de dificultades y esquemas conceptuales en la enseñanza de la Química.Enseñanza de las Ciencias, 4, 1, pp. 73-77.

Furió, C. y Ortiz, E. (1983) Persistencia de errores conceptuales en el estudio del equilibrio químico. Enseñanza de las Ciencias, 1, pp. 15-20.

Furió, C., Hernández, J. y Harris, H. (1987) Parallels between adolescents' conception of gases and the history of Chemistry.Journal of Chemical Education, 64, 7, pp. 617-618.

Geertz, C. (1988) La interpretación de las culturas (Barcelona, Gedisa). EUMC

Gil, D. (1982) La investigación en el aula de Física y Química . Madrid, Anaya.

Gil, D. (1987) Los errores conceptuales como origen de un nuevo modelo didáctico: de la búsqueda a la investigación. Investigación en la Escuela, 1, pp. 35-41.

Gil, D. y Payá, J. (1988) Los trabajos prácticos de Física y Química y la metodología científica. Enseñanza de la Física, 2, 2, pp. 73-79.

Gil, D., Carrascosa, J., Furió, C. y Martínez-Torregrosa, J. (1991) La enseñanza de las ciencias en la Educación Secundaria. Barcelona, ICEHorsori.

Gil, D., Dumas-Carre, A., Caillot, M. y Martínez-Torregrosa, J. (1989) La resolución de problemas de lápiz y papel como actividad de investigación. Investigación en la Escuela, 6, pp. 3-20.

Gimeno, J. (1981) Teoría de la enseñanza y desarrollo del curriculo. Madrid, Anaya.

Gimeno, J. (1982) La pedagogía por objetivos. Obsesión por la eficacia. Madrid, Morata.

Gimeno, J. y Pérez, A.I. (1992) Comprender y transformar la enseñanza. Madrid, Morata.

Giordan, A. y Vecchi, G. de (1988) Los orígenes del saber: de las concepciones personales a los conceptos científicos. Sevilla, Diada.

Gutiérrez, R. (1987) Psicología y aprendizaje de las ciencias. El modelo de Ausubel. Enseñanza de las Ciencias, 5, 2, pp. 118-128.

Hernández, A. (1989) Cultura y desarrollo en el medio rural Madrid, Narcea. 
Hewson, P. W. y Hewson, M.G. (1987) Science teachers' conceptions of teaching: implications for teachers' educations. International Journal of Science Education, 9, 4, pp. 425-440.

Hewson, P.W. y Hewson, M.G. (1988) On appropriate conception of teaching: implications for teachers educations. International Journal of Science Education, 72, 5, pp. 597-614.

Hierrezuelo, J. y Montero, A. (1989) La ciencia de los alumnos: su utilización en la didáctica de la Física y Química . Madrid, Laia-MEC.

Jean, G. (1982) El profesor: su cultura personal y su acción pedagógica Madrid, Narcea.

Johnston, K y Scott, P. (1991) Diagnostic teaching in the classroom: teaching/learning strategies to promote development in understanding about conservation of mass on dissolving. Research in Science and Technological Education, 9, 2, pp. 193-212.

Krathwohl, D. R. (ed.) (1973) Taxonomía de los objetivos de la educación. Clasificación de las metas educativas. Ambito de la afectividad. Alcoy, Marfil.

Levine, R. y White, M. (1986) El hecho humano. Las bases culturales del desarrollo educativo . Madrid, Visor/MEC.

Lobrot, M. (1976) La Pedagogía Institucional. Buenos Aires, Humanitas.

Llorens, J.A. (1989) El proceso de cambio conceptual en la iniciación de la Química. La introducción de los conceptos de sustancia pura y cambio químico. Revista de Educación, 289, pp. 307-332.

Llorens, J.A., Llopis, R. y Jaime, M.C. de (1987) El uso de la terminología científica en los alumnos que comienzan el estudio de la Química en la enseñanza media. Una propuesta metodológica para su análisis. Enseñanza de las Ciencias, 5, 1, pp. 33-40.

Marco, B. et al. (1987) La enseñanza de las ciencias experimentales. Proyecto 12-16. Madrid, Narcea.

Martínez-Torregrosa, J. et al. (1991)Programas-guía de Física y Química para el ciclo 12-14 de la Educación Secundaria. Documentos de trabajo . Alicante, CEP de Alicante.

Neisser, U. (1987) Concepts and conceptual development. Cambridge, Cambridge University Press.

Novak, J.D. y Gowin, A. (1989) Aprender a aprender . Barcelona, Martínez Roca.

Pérez Gómez, A.I.(1988) Curriculum y enseñanza: Análisis de componentes. 
Málaga, Universidad de Málaga.

Pérez Gómez, A.I. (1992) Una escuela para recrear la cultura. Cuadernos de Pedagogía, 207, octubre, pp. 48-54.

Pérez Gómez, A.I. (1998) La cultura institucional de la escuela.Cuadernos de Pedagogía, 266, febrero, pp. 79-82

Peters, R.S. (1977) Filosofía de la Educación. México, Fondo de Cultura Económica.

Posada, J.M. de (1993) Concepciones de los alumnos de 15-18 años sobre la estructura interna de la materia en estado sólido. Enseñanza de las Ciencias, 11,1, pp. 12-19.

Posada, J.M. de y Prieto, T. (1989) Ideas y representaciones de los alumnos sobre radiactividad. Revista de Educación, 289, pp. 357-375.

Posada, J.M. de y Prieto, T. (1990) Exploraciones gráficas de ideas extraescolares de los alumnos sobre radiactividad. Enseñanza de las Ciencias, 8, 2, pp. 127-130.

Pozo, J.I. (1987) Aprendizaje de la ciencia y pensamiento causal . Madrid, Visor.

Pozo, J.I. (1989) Teorías cognitivas del aprendizaje . Madrid, Morata.

Pozo, J.I. (1990) Una nueva forma de aprender. Cuadernos de Pedagogía, 180, pp. 24-27.

Pozo, J.I., Gómez, M.A., Limón, M. y Sanz, A. (1991) Procesos cognitivos en la comprensión de la ciencia: las ideas de los adolescentes sobre la Química. Madrid, MEC.

Ramírez, L. (1990) La resolución de problemas de Física y Química como investigación en la enseñanza media, un instrumento de cambio metodológico. Universidad de Barcelona, Facultad de Ciencias Químicas.

Ross, B. et al. (1991) Concept mapping and misconceptions: a study of highschool students' understandings of acids and bases. International Journal of Science Education, 13, 1, pp. 11-24.

Sarason, S.B. (1982) The culture of the school and the problem of change Boston, Allyn and Bacon.

Sebastiá, J.M. (1989) El constructivismo: un marco teórico problemático. Enseñanza de las Ciencias, 7, 2, pp. 158-161.

Serrano, T. (1988) Actitudes de los alumnos y aprendizaje de las ciencias: un estudio longitudinal. Investigación en la Escuela, 5, pp. 29-38. 
Shayer, M. y Adey, P. (1984) La ciencia de enseñar ciencias . Madrid, Narcea.

Snow, C.P. (1977) Las dos culturas y un segundo enfoque Madrid, Alianza.

Stavy, R. (1991) Children's ideas about matter. School Science and Mathematics, 91, 6, pp. 240-244.

Torre, S. de la (1993) Aprender de los errores . Madrid, Escuela Española.

Wertsch, J.V. (1985) Culture, communication and cognition.Vygotskian perspectives Cambridge, University Press.

Westoby, A. (1988) Culture and Power in educational organizations. Philadelphia, Open University Press.

(C) Ediciones Universidad de Salamanca.

Páginas mantenidas por Francisco Ignacio Revuelta Domínguez

y Luis González Rodero

webmrte@usal.es 